\title{
Low-Q Electrically Small Spherical Magnetic Dipole Antennas
}

\author{
Kim, Oleksiy S.
}

Published in:

I E E E Transactions on Antennas and Propagation

Link to article, DOI:

10.1109/TAP.2010.2048863

Publication date:

2010

Document Version

Early version, also known as pre-print

Link back to DTU Orbit

Citation (APA):

Kim, O. S. (2010). Low-Q Electrically Small Spherical Magnetic Dipole Antennas. I E E E Transactions on Antennas and Propagation, 58(7), 2210-2217. https://doi.org/10.1109/TAP.2010.2048863

\section{General rights}

Copyright and moral rights for the publications made accessible in the public portal are retained by the authors and/or other copyright owners and it is a condition of accessing publications that users recognise and abide by the legal requirements associated with these rights.

- Users may download and print one copy of any publication from the public portal for the purpose of private study or research.

- You may not further distribute the material or use it for any profit-making activity or commercial gain

- You may freely distribute the URL identifying the publication in the public portal

If you believe that this document breaches copyright please contact us providing details, and we will remove access to the work immediately and investigate your claim. 


\title{
Low-Q Electrically Small Spherical Magnetic Dipole Antennas
}

\author{
Oleksiy S. Kim
}

\begin{abstract}
Three novel electrically small antenna configurations radiating a $\mathrm{TE}_{10}$ spherical mode corresponding to a magnetic dipole are presented and investigated: multiarm spherical helix (MSH) antenna, spherical split ring resonator (S-SRR) antenna, and spherical split ring (SSR) antenna. All three antennas are self-resonant, with the input resistance tuned to $50 \mathrm{ohms}$ by an excitation curved dipole/monopole. A prototype of the SSR antenna has been fabricated and measured, yielding results that are consistent with the numerical simulations. Radiation quality factors $(Q)$ of these electrically small antennas (in all cases $k a<0.26$ ) approach the limit of 3.0 times the Chu lower bound for a given antenna size, which is in line with a theoretical prediction made by Wheeler in 1958.
\end{abstract}

Index Terms-Electrically small antennas, magnetic dipole, Chu limit, quality factor, split-ring resonator (SRR), surface integral equation

\section{INTRODUCTION}

$\mathbf{F}$ UNDAMENTAL electromagnetic properties of electrically small antennas (ESA) were first explored by Wheeler [1] and Chu [2] more than 60 years ago. Whereas Wheeler focuses on limitations peculiar to electrically small electric and magnetic dipole antennas, Chu develops a more sophisticated theory based on spherical TM and TE modes radiated by a generalized omnidirectional ESA. Subsequently revisited and corrected by a number of authors [3]-[6], Chu's theory establishes a lower bound for a radiation quality factor $Q$ achievable by a small antenna occupying a certain volume. The quality factor $Q$ relates the reactive energy to the radiated power, and for a single-resonance ESA it is inversely proportional to the antenna frequency bandwidth. Chu assumes that the reactive electric (magnetic) energy is stored entirely outside a minimum sphere of radius $a$ and thus derives an ultimate lower bound $Q_{\mathrm{LB}}$ for an electrically small TM-mode (TE-mode) antenna. Any passive antenna inscribed in a sphere of radius a cannot perform better than that. More realistic configurations are considered by Thal in [7], where he starts with an impressed electric current on a spherical surface and allows the field not only outside but also inside the sphere to satisfy the boundary condition. It is not surprising that Thal's bounds are higher than those by Chu. For instance, Thal shows that for an electric dipole $\left(\mathrm{TM}_{10}\right.$ mode) antenna $Q \rightarrow 1.5 Q_{\mathrm{LB}}$ as $k a \rightarrow 0$, where $k$ is the free space propagation constant. This result is confirmed by simulations and measurements of real antennas designed by Best [8], [9].

This work is supported by the Danish Research Council for Technology and Production Sciences within the TopAnt project (http://www.topant.dtu.dk).

The author is with the Department of Electrical Engineering, Electromagnetic Systems, Technical University of Denmark, DK-2800 Kgs. Lyngby, Denmark (e-mail: osk@elektro.dtu.dk)

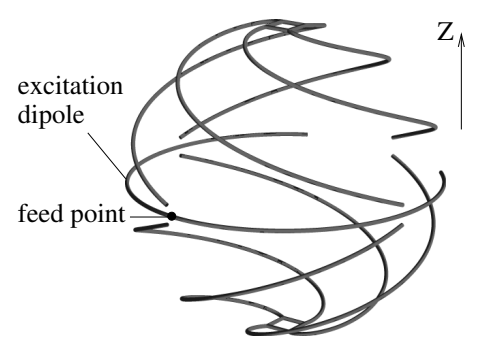

(a)

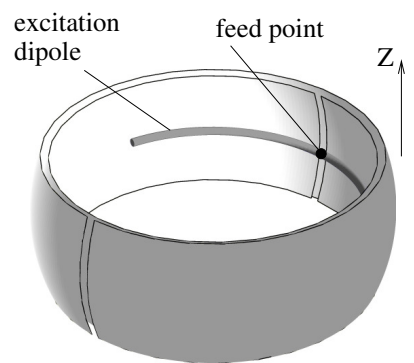

(b)

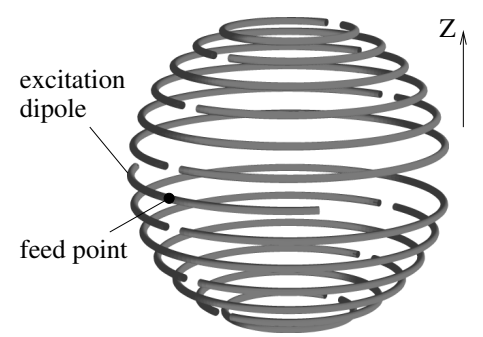

(c)

Fig. 1. Geometries of spherical magnetic dipole ( $\mathrm{TE}_{10}$ mode) antennas: (a) Multiarm spherical helix (MSH) antenna; (b) spherical split-ring resonator (SSRR) antenna; (c) spherical split ring (SSR) antenna.

What is more interesting is that Thal's bounds for electric and magnetic dipole antennas are not equal. According to Thal, for an electrically small magnetic dipole ( $\mathrm{TE}_{10}$ mode) antenna $Q=3.0 Q_{\mathrm{LB}}$. In fact, the latter result was also predicted by Wheeler in 1958 [10]. Recently, a 16-slot electrically small $\mathrm{TE}_{10}$-mode antenna yielding the quality factor $Q=3.18 Q_{\mathrm{LB}}$ was reported by Best [11].

This paper presents three novel electrically small antenna configurations (Fig. 1) designed to radiate the $\mathrm{TE}_{10}$ spherical mode corresponding to a magnetic dipole (z-directed in Fig. 1). These antennas are a multiarm spherical helix (MSH) antenna, a spherical split-ring resonator (S-SRR) antenna, and a spherical split ring (SSR) antenna. Being quite different in geometry, all three antennas are self-resonant with no external lumped elements used to ensure the resonance. Basically, each antenna 
is a spherical resonator excited by a curved dipole, whose length is varied appropriately to match the antenna to a feed line in a way similar to [12], [13]. The $\mathrm{TE}_{10}$-mode antennas presented in this paper not only validate the Wheeler-Thal lower bound for magnetic dipole antennas, but also provide a necessary basis for designing low- $Q$ ESA that outperform electric dipole antennas. As shown in [14], a magnetic dipole antenna augmented with a magnetic core yields the quality factor $Q$ approaching the Chu lower bound.

In this paper, numerical results are obtained using a surface integral equation (SIE) technique combined with a higherorder method of moments [15]. The antennas are assumed to be lossless with a delta-gap generator as a signal source. The quality factor $Q$ is calculated from the antenna input impedance $Z(\omega)=R(\omega)+j X(\omega)$ as [16]

$$
Q \approx \frac{\omega_{0}}{2 R\left(\omega_{0}\right)}\left|Z^{\prime}\left(\omega_{0}\right)\right|
$$

where $\omega_{0}$ is the angular frequency at the resonance.

In terms of comparing the quality factors of practical antennas with the lower bound, it is important to recognize that the presence of higher-order modes affects the quality factor, and this effect must be accounted for not only in the calculations of the antenna $Q$ itself, but also in the bound it is compared with. In the next section of this paper, a brief note on the evaluation of the quality factor lower bound for a magnetic dipole antenna in the presence of higher-order modes $\left(Q_{\mathrm{LB}}^{\mathrm{HO}}\right)$ is given.

The next three sections contain results of parametric investigations for each of the three antenna configurations, respectively. It is shown how the bound $Q=3.0 Q_{\mathrm{LB}}$ is approached by changing appropriate antenna geometry parameters as well as how the resonance frequency and the input resistance at resonance are tuned to the desired values. Experimental results for a fabricated SSR antenna are also presented.

\section{QUALity FACTOR LOWER BOUND AND HIGHER-ORDER MODES}

The quality factor lower bound for an electrically small magnetic dipole non-resonant antenna tuned to a resonance by a lossless reactive element and/or by distributed reactive fields is given by [6]

$$
Q_{\mathrm{LB}}=\frac{2 \omega W_{0}^{m}}{P_{0}^{m}}=\frac{1}{(k a)^{3}}+\frac{1}{k a}
$$

where $W_{0}^{m}$ and $P_{0}^{m}$ are the time-average stored magnetic energy external to the antenna sphere of radius $a$ and the radiated power of the magnetic dipole mode $\left(\mathrm{TE}_{10}\right.$ spherical mode), respectively. In the presence of higher-order modes the bound $Q_{\mathrm{LB}}^{\mathrm{HO}}$ can be exactly computed using expressions provided by Fante [5]. However, this requires knowledge of the whole spectrum of spherical modes radiated by an antenna.

In many cases, a somewhat easier approach can be undertaken. To account for higher-order modes the expression (2) is modified as

$$
\begin{aligned}
Q_{\mathrm{LB}}^{\mathrm{HO}} & =\frac{2 \omega \max \left\{W^{m}, W^{e}\right\}}{P}=\frac{2 \omega W^{m}}{P} \\
& =\frac{2 \omega\left(W_{0}^{m}+W_{\mathrm{HO}}^{m}\right)}{\left(P_{0}^{m}+P_{\mathrm{HO}}^{m}+P_{\mathrm{HO}}^{e}\right)}
\end{aligned}
$$

where $W_{\mathrm{HO}}^{m}$ and $P_{\mathrm{HO}}^{m}$ are the external stored magnetic energy and the radiated power of higher-order magnetic modes (TE spherical modes), respectively. For an electrically small magnetic dipole antenna, besides the radiated power $P_{\mathrm{HO}}^{e}$, the higher-order TM modes contribute predominantly to the stored electric energy, and thus negligibly change the numerator in (3). On the other hand, the stored magnetic energy is defined by the fundamental $\mathrm{TE}_{10}$ mode as well as by the higher-order TE modes that, unlike the higher-order TM modes, increase the quality factor [5], and therefore must be suppressed in a well-designed magnetic dipole antenna. In this case, the bound (3) can be simplified as

$$
Q_{\mathrm{LB}}^{\mathrm{HO}}=\frac{2 \omega W_{0}^{m}}{\left(P_{0}^{m}+P_{\mathrm{HO}}^{e}\right)}=\frac{2 \omega W_{0}^{m}}{P}=K_{0}^{m} Q_{\mathrm{LB}}
$$

where $K_{0}^{m}=P_{0}^{m} / P$ is the power of the $\mathrm{TE}_{10}$ mode relative to the total radiated power. Thus, to compute the lower bound $Q_{\mathrm{LB}}^{\mathrm{HO}}$ it is sufficient to evaluate a single spectrum component - the amplitude of the $\mathrm{TE}_{10}$ mode.

The expression (4) for a magnetic dipole $\mathrm{TE}_{10}$ mode antenna is valid under three conditions:

1) the antenna is electrically small, so that TM modes contribute negligibly to the stored magnetic energy;

2) higher-order TE modes are suppressed enough to be neglected;

3) externally to the antenna, the stored magnetic energy exceeds the stored electric energy $\left(W^{m}>W^{e}\right)$.

As shown below, all antennas presented in this paper fulfill the first two conditions, whereas the third one is only met by the S-SRR and SSR antennas. The third condition is violated by some magnetic dipole antennas, in which due to higherorder TM modes the stored electric energy outside the antenna is superior to the corresponding stored magnetic energy. If such an antenna is self-resonant, e.g., the MSH antenna, the overall energy balance is restored by the surplus of the stored magnetic energy inside the antenna. In this case, an expression dual to (3a) must be applied, although the radiated power is by far dominated by the main $\mathrm{TE}_{10}$ mode.

It is noted, that the considerations presented in this section are by duality applicable for electric dipole antennas.

\section{Multiarm Spherical Helix (MSH) Antenna}

The $\mathrm{TE}_{10} \mathrm{MSH}$ antenna is a modification of a spherical helix antenna developed by Best [9]. The original configuration (Fig. 2a) radiates the $\mathrm{TM}_{10}$ spherical mode, since the driving voltage is applied vertically in the middle of one of the arms. Due to the symmetry and small electrical dimensions, the far-field contributions from the $\phi$-components of the electric current on the wires $\left(I_{\phi}\right)$ mutually cancel, thus leaving only the $\theta$-component of the electric far-field (and $\phi$ component of the magnetic far-field).

In the $\mathrm{TE}_{10} \mathrm{MSH}$ antenna, top and bottom parts of the arms are disconnected, and a curved dipole is placed at the equator of the antenna sphere (Fig. 2b). The driving voltage is now applied horizontally at the midpoint of the excitation dipole. In this case, the far-field contributions from the $\theta$-components of the electric current $\left(I_{\theta}\right)$ cancel, and the resulting radiated fields are those of the desired $\mathrm{TE}_{10}$ spherical mode. 


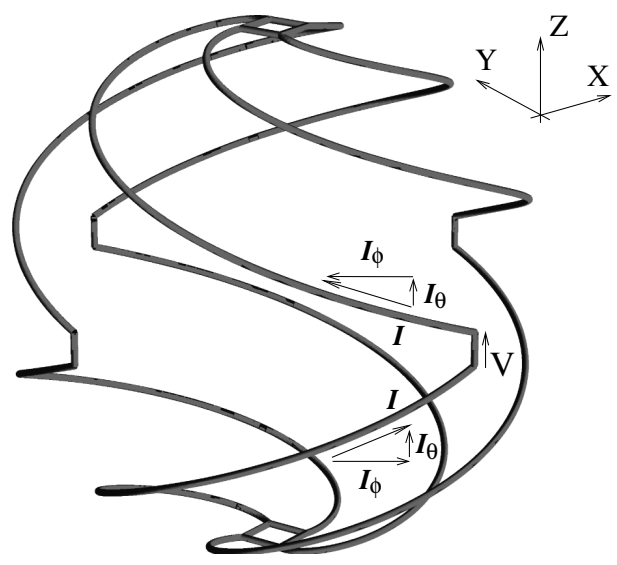

(a)

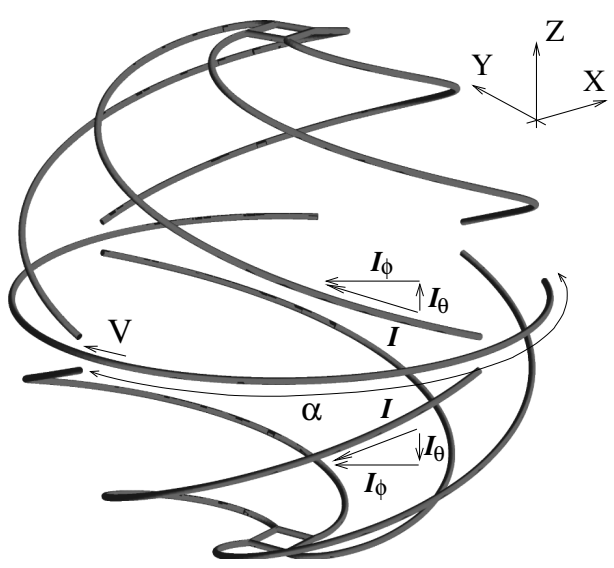

(b)

Fig. 2. Multiarm spherical helix (MSH) antenna: (a) $\mathrm{TM}_{10}$ (electric dipole); (b) $\mathrm{TE}_{10}$ (magnetic dipole).

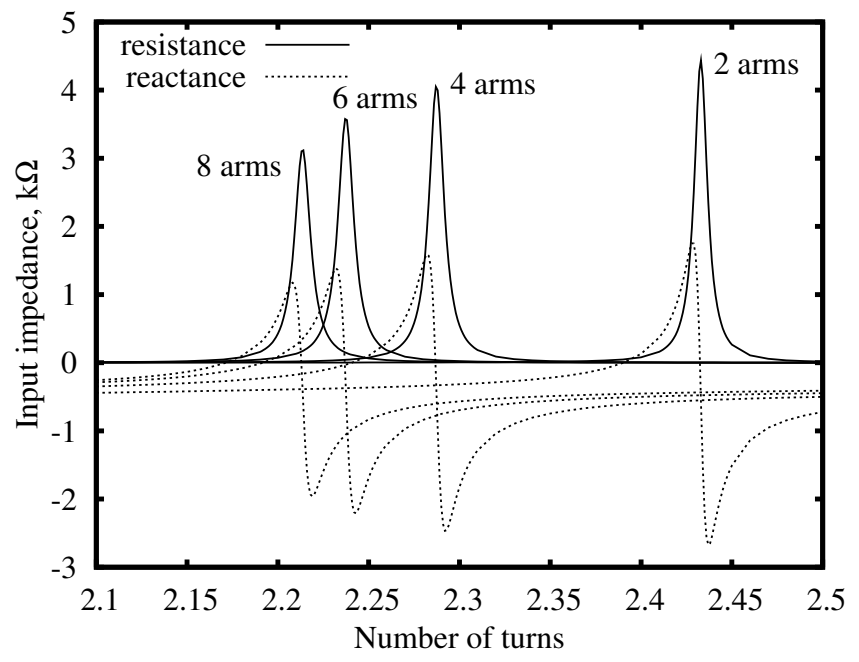

Fig. 3. Input impedance of the $\mathrm{TE}_{10} \mathrm{MSH}$ antenna as a function of the number of turns in each arm.

For a given frequency $f_{0}$ and number of arms $\left(N_{\text {arms }}\right)$, the antenna is tuned to the resonance by changing the number of turns $\left(N_{\text {turns }}\right)$ in the arms as shown in Fig. 3, where the antenna input impedance at $f_{0}=300 \mathrm{MHz}$ is plotted for four antenna configurations having $N_{\text {arms }}=2,4,6$, and 8 arms. All antenna configurations have radius $r_{0}=40 \mathrm{~mm}$,

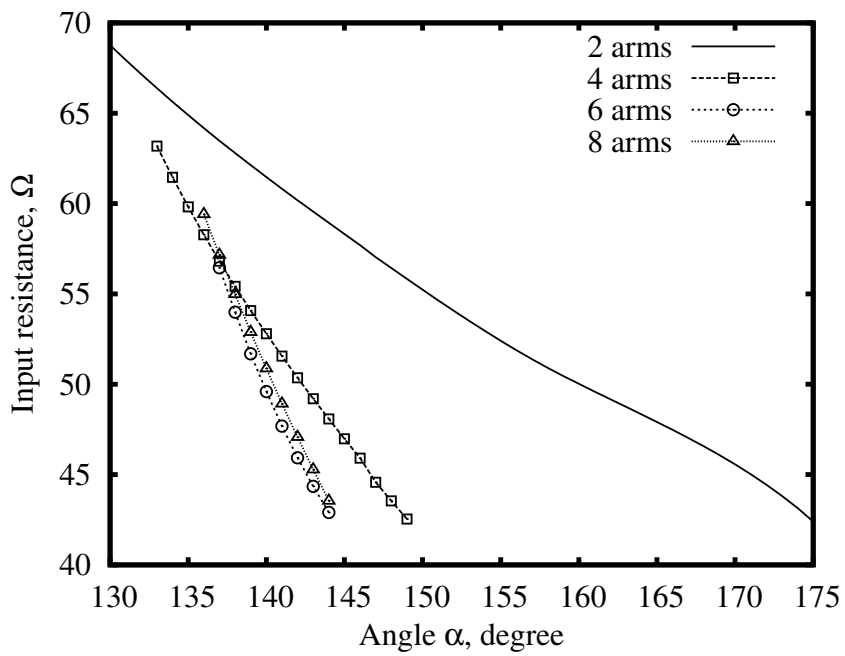

(a)

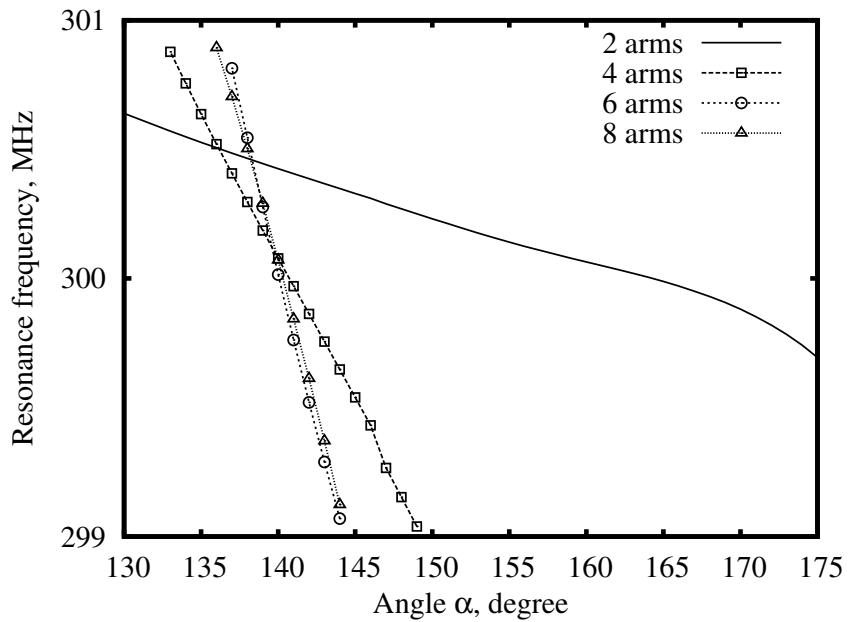

(b)

Fig. 4. Input resistance at resonance (a) and the resonance frequency (b) of the $\mathrm{TE}_{10} \mathrm{MSH}$ antenna as a function of the excitation dipole length.

and the wire radius is set to $0.5 \mathrm{~mm}$. Thus, the antennas occupy a spherical volume of radius $a=40.5 \mathrm{~mm}$, or $k a=0.254$ at $300 \mathrm{MHz}$. Observing Fig. 3 one may note that the quality factor decreases as the number of arms multiplied. From Table I, which summarizes the geometry parameters for self-resonant antenna configurations and corresponding quality factors $Q$, it is seen that the quality factor indeed decreases approaching $Q=3.0 Q_{\mathrm{LB}}$.

Besides the number of arms and the number of turns in each arm, there is another important antenna geometry parameter - the length of the curved excitation dipole. In this paper, it is quantified in angular units and denoted by $\alpha$ (see Fig. 2b). It controls the antenna input resistance at resonance $R_{0}$ and makes matching of the antenna to external circuits a fairly straightforward task. For instance, the results presented in Fig. 3 and Table I, are obtained with the length $(\alpha)$ chosen so that for each number of arms the antenna is matched to $50 \mathrm{ohms}$ at the resonance. Figure 4a shows the dependence of the antenna input resistance $R_{0}$ on the angle $\alpha$ with the number of arms as a parameter. For a fixed number of turns (see Table I for the values), a change in the length of the excitation dipole 
TABLE I

CHARACTERISTICS OF THE TE 10 MSH ANTENNA.

\begin{tabular}{ccccccccccc}
\hline$N_{\text {arms }}$ & $N_{\text {turns }}$ & $\alpha,{ }^{\circ}$ & $Q$ & $Q_{\mathrm{LB}}$ & $Q / Q_{\mathrm{LB}}$ & $\mathrm{TE}_{10}, \mathrm{~dB}$ & $\mathrm{TM}_{11}, \mathrm{~dB}$ & $\mathrm{TM}_{20}, \mathrm{~dB}$ & $Q_{\mathrm{LB}}^{\mathrm{HO}}$ & $Q / Q_{\mathrm{LB}}^{\mathrm{HO}}$ \\
\hline 2 & 2.39 & 163 & 249.6 & 64.6 & 3.87 & -0.047 & -22.4 & -23.0 & 91.1 & 2.74 \\
4 & 2.24 & 143 & 218.5 & 64.6 & 3.39 & -0.030 & -27.2 & -23.0 & 90.6 & 2.41 \\
6 & 2.19 & 140 & 210.7 & 64.6 & 3.27 & -0.027 & -29.5 & -23.0 & 90.0 & 2.34 \\
8 & 2.17 & 140 & 207.0 & 64.6 & 3.21 & -0.026 & -30.4 & -23.0 & 90.0 & 2.30 \\
\hline
\end{tabular}

causes a little shift in the resonance frequency as depicted in Fig. 4b. By adjusting the number of turns the deviation in the resonance frequency is easily compensated.

The presented antenna is electrically small and its radiation pattern closely reproduces that of an elementary magnetic dipole with the directivity $1.76 \mathrm{~dB}$. A spherical wave expansion of the antenna far-fields shows that next most significant spherical modes after the fundamental $\mathrm{TE}_{10}$ mode are $\mathrm{TM}_{11}$ and $\mathrm{TM}_{20}$. The radiated power of these modes normalized to the total radiated power is given in Table I. As the number of arms increases, the relative radiated power of the $\mathrm{TM}_{11}$ mode decreases, whereas the $\mathrm{TM}_{20}$ mode stays constant at a level, which is so high that the stored electric energy external to the antenna exceeds the external stored magnetic energy. Since the antenna is self-resonant, the energy balance is by necessity recovered by the opposite energy difference inside the antenna. However, the lower bound $Q_{\mathrm{LB}}^{\mathrm{HO}}$ must now be computed using the stored electric energy, and this is done by applying the exact expressions [5, eq.(7)]. As shown in Table I, due to the presence of the relatively strong $\mathrm{TM}_{20}$ mode the lowest achievable quality factor is noticeably higher than that of the $\mathrm{TE}_{10}$ mode alone $\left(Q_{\mathrm{LB}}\right)$. Consequently, the ratio $Q / Q_{\mathrm{LB}}^{\mathrm{HO}}$ becomes less than 3.0.

\section{SPHericAl SPlit-RING Resonator (S-SRR) ANTENNA}

The S-SRR antenna consists of a spherical split-ring resonator and a curved excitation dipole (Fig. 1b) arranged so that mostly $\phi$-directed electric surface currents of the desired $\mathrm{TE}_{10}$ mode are excited. Unlike the previous case, a symmetry along the XZ-plane allows the antenna to be placed on a metal ground plane as sketched in Fig. 5. The S-SRR is a conformal to a sphere variation of a broadside coupled splitring resonator [17], [18]. Due to a large overlapping area of two spherical surfaces the S-SRR can be easily made electrically small. Results below are presented for the S-SRR antenna on an infinite perfectly electrically conducting (PEC) ground plane; and the geometrical parameters are as follows: $r_{0}=a=21 \mathrm{~mm}, t=0.8 \mathrm{~mm}, g=1 \mathrm{~mm}, r_{\mathrm{mnp}}=18 \mathrm{~mm}$, and the radius of the monopole is $0.5 \mathrm{~mm}$.

In terms of low $Q$ it is desirable to distribute the electric current over the entire spherical surface of radius $r_{0}=a$, that is to allow the S-SRR to cover the whole sphere $(\beta \rightarrow \pi)$. However, this blocks the magnetic flux over the antenna crossection and thus diminishes the electric current on the antenna surface. Therefore, there should be expected an optimal coverage $\left(\beta=\beta_{0}\right)$ yielding a minimum $Q$. Indeed, the dependence of the ratio $Q / Q_{\mathrm{LB}}$ on the angle $\beta$ plotted in Fig. 6a exhibits an optimum at $\beta=73^{\circ}$.

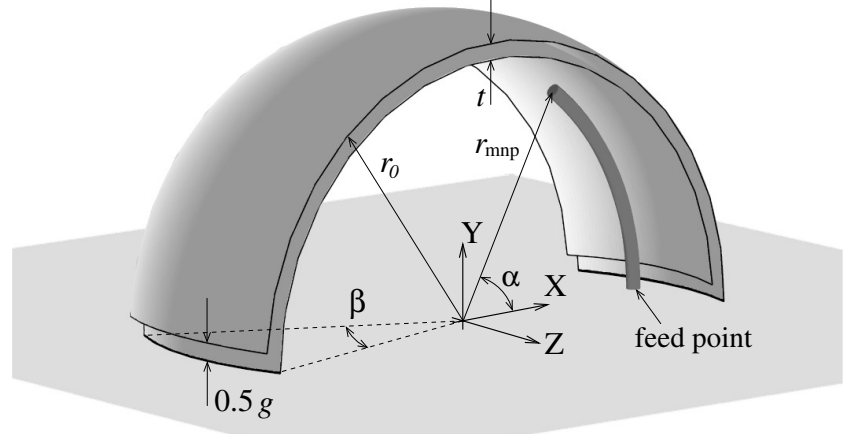

Fig. 5. Spherical split-ring resonator (S-SRR) antenna on a ground plane.

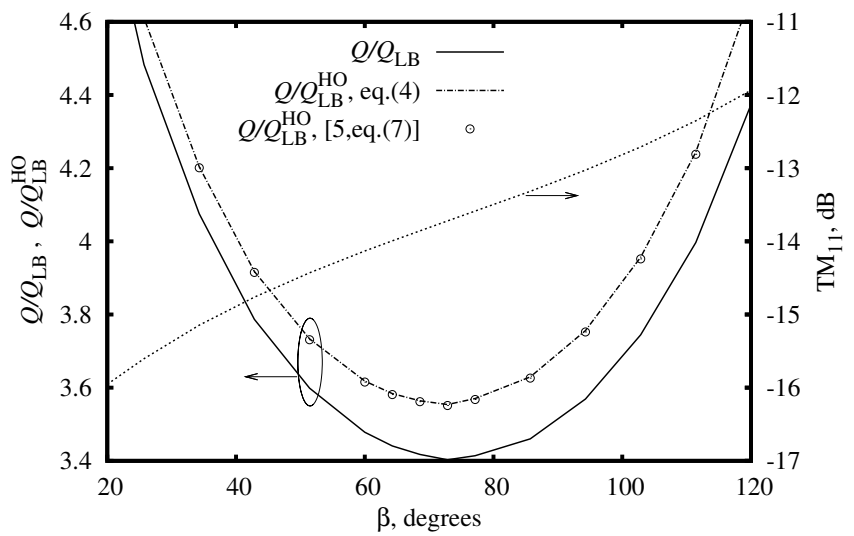

(a)

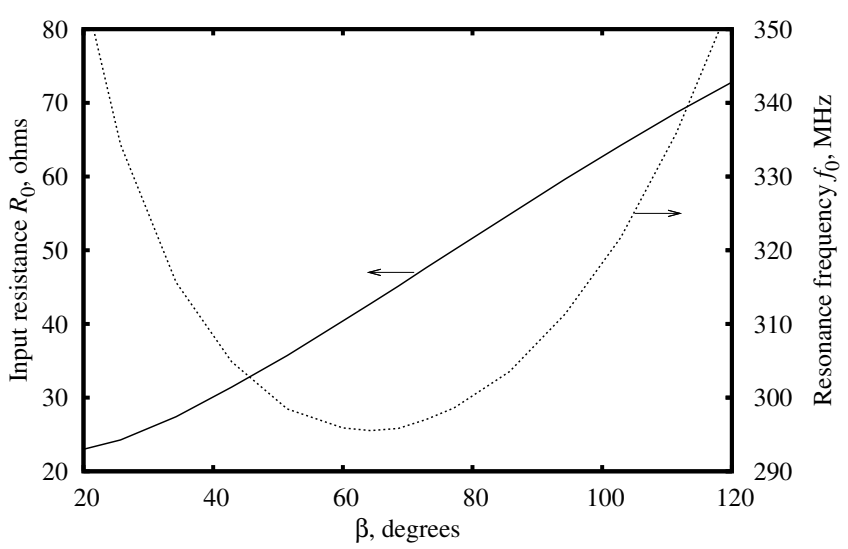

(b)

Fig. 6. Properties of the S-SRR antenna as a function of angle $\beta$ : (a) ratio $Q / Q_{\mathrm{LB}}$ and the relative radiated power of the $\mathrm{TM}_{11}$ mode; (b) input resistance at resonance and the resonance frequency.

The resonance frequency variation versus $\beta$ (Fig. 6b) shows the similar behavior yielding minimum at $\beta=64^{\circ}$. Although the location of this minimum does not coincide with 


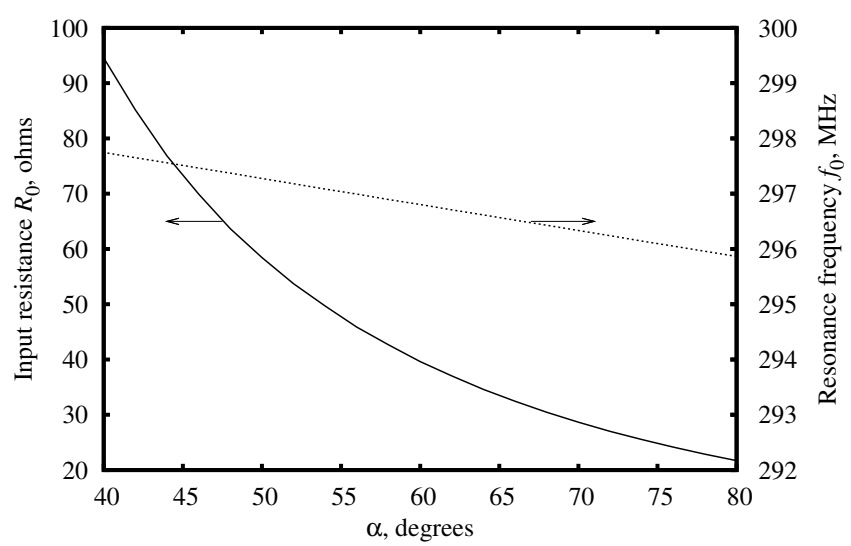

Fig. 7. Input resistance at resonance and the resonance frequency of the S-SRR antenna as a function of the excitation monopole length.

the optimum $\beta$ for the ratio $Q / Q_{\mathrm{LB}}$, the difference in the resonance frequency for $\beta=64^{\circ}$ and $\beta=73^{\circ}$ is minor, being only $1.5 \mathrm{MHz}$. For $\beta=73^{\circ}$ the resonance frequency is $f_{0}=297.0 \mathrm{MHz}$, and the electrical size of the antenna is $k a=0.133$.

Fig. $6 \mathrm{~b}$ also illustrates the dependence of the antenna input resistance $R_{0}$ at resonance on the angle $\beta$. For this plot as well as for the simulations above, the length of the excitation monopole, which likewise the excitation dipole in the MSH antenna (Section III) controls the input impedance, is chosen to be $\alpha=55^{\circ}$, so that $R_{0}=50 \mathrm{ohms}$ for the optimal $\beta=73^{\circ}$.

In the spherical wave spectrum of the antenna, $\mathrm{TM}_{11}$ is the only most significant higher-order mode, whereas $\mathrm{TM}_{20}$ - the strongest higher-order mode in the spectrum of the MSH antenna (Section III) - in this case is totally suppressed due to absence of $\theta$-directed currents in the XZ-plane. The $\mathrm{TM}_{11}$ mode is however very pronounced and thus noticeably influences the ratio $Q / Q_{\mathrm{LB}}^{\mathrm{HO}}$, as shown in Fig. 6a. The $\mathrm{S}$ SRR antenna fulfills all three conditions in Section II, and therefore, the expression (4) is applied to compute the lower bound $Q_{\mathrm{LB}}^{\mathrm{HO}}$. An excellent agreement with the ratio obtained using the exact expression [5, eq.(7)] validates the approach introduced in Section II.

The tuning properties of the S-SRR antenna are demonstrated in Fig. 7, where the resonance frequency $f_{0}$ and the input resistance at resonance $R_{0}$ are plotted versus the length of the monopole for a fixed $\beta=73^{\circ}$. A weak variation of the resonance frequency is observed, while a broad variation of the input resistance allows the antenna to be matched to a wide range of feed lines.

In summary, the minimum ratio $Q / Q_{\mathrm{LB}}$ exhibited by the $\mathrm{S}$ SRR antenna is close to that of the 4-arm MSH antenna (Section III) On the other hand, the symmetry in the XZ plane eliminates the $\mathrm{TM}_{20}$ mode and enables the S-SRR antenna to operate on a ground plane.

\section{Spherical Split Ring (SSR) Antenna}

In this novel antenna configuration, the spherical resonator is composed of individual wire split rings distributed evenly in $\theta$ (Fig. 1c). Every two neighbor rings are flipped with respect

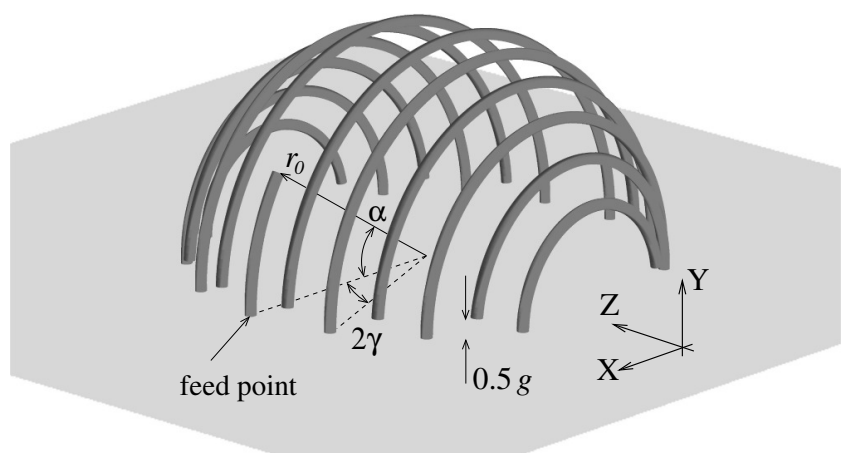

Fig. 8. Spherical split ring (SSR) antenna on a ground plane.

to each other and, thus, operate as a conventional SRR. Combined with other rings they constitute a multielement SRR. This arrangement ensures a more uniform current distribution over the antenna spherical surface as well as a great reduction of the resonance frequency as compared to a single twoelement SRR and, a fortiori, a single split ring. The number of the rings are chosen to be odd, so that the central split ring serves as an excitation dipole with arm lengths adjusted to match the antenna to a feed line. As in the previous case, the SSR antenna possesses the symmetry in the XZ-plane and, consequently, an ability to operate on a ground plane (Fig. 8).

For a given radius of the spherical resonator $r_{0}$, the resonance frequency is essentially controlled by the number of split rings $N_{\mathrm{SR}}$; finer adjustments can be made via changing the gap width $g$ in the rings. The number of split rings is determined using the following expression

$$
N_{\mathrm{SR}}=2 \operatorname{int}\left\{\frac{90^{\circ}}{\gamma}\right\}-1
$$

where $\gamma$ is an angular separation between two neighbor rings (Fig. 8).

\section{A. Numerical Results}

Here, results are presented for the SSR antenna on an infinite PEC ground plane. Some of the geometrical parameters are fixed as follows: $r_{0}=21 \mathrm{~mm}, g=2 \mathrm{~mm}$, and the radius of the wires is $0.5 \mathrm{~mm}$. The separation angle $\gamma$ is varied to elucidate the dependence of the antenna quality factor on the number of split rings. The result presented in Fig. 9a shows that as the split rings get closer to each other and their number increases, the ratio $Q / Q_{\mathrm{LB}}$ monotonically decreases. And so does the relative radiated power of the parasitic $\mathrm{TM}_{11}$ mode. As compared to the S-SRR antenna (Section IV), its level is highly reduced, and its influence on the $Q / Q_{\mathrm{LB}}^{\mathrm{HO}}$ ratio is minor (Fig. 9a). Again, the $\mathrm{TM}_{20}$ mode is totally suppressed due to absence of $\theta$-directed wires.

Variations of the resonance frequency $f_{0}$ and the input resistance at resonance $R_{0}$ versus the angle $\gamma$ are plotted in Fig. 9b. In these simulations the length of the excitation monopole (the central split ring) is fixed to $\alpha=30^{\circ}$, which yields $R_{0}=50 \mathrm{ohms}$ input impedance for $\gamma=4.9^{\circ}$, or $N_{\mathrm{SR}}=35$. If $\alpha$ is varied, it changes the input resistance $R_{0}$ as illustrated in Fig. 10. Corresponding changes in the resonance frequency $f_{0}$ also shown in Fig. 10 are within $\pm 1 \%$. 


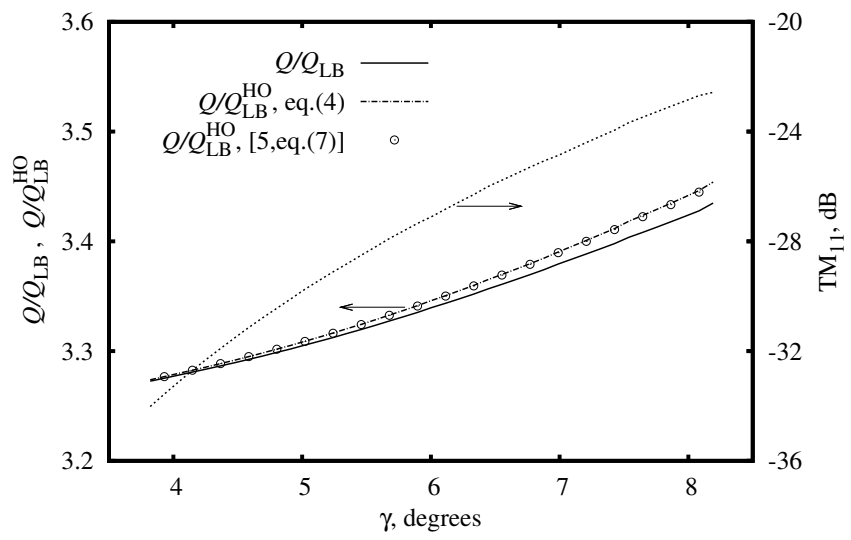

(a)

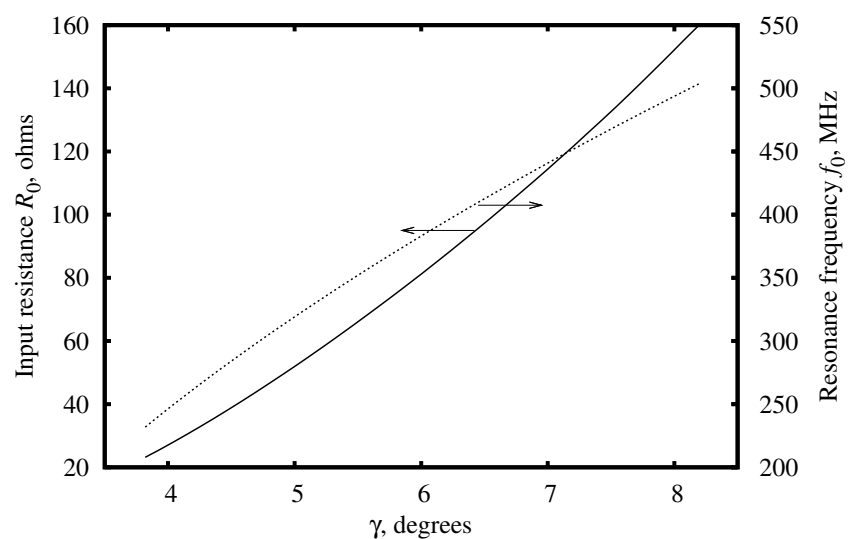

(b)

Fig. 9. Properties of the SSR antenna as a function of angle $\gamma$ : (a) ratio $Q / Q_{\mathrm{LB}}$ and the relative radiated power of the $\mathrm{TM}_{11}$ mode; (b) input resistance at resonance and the resonance frequency.

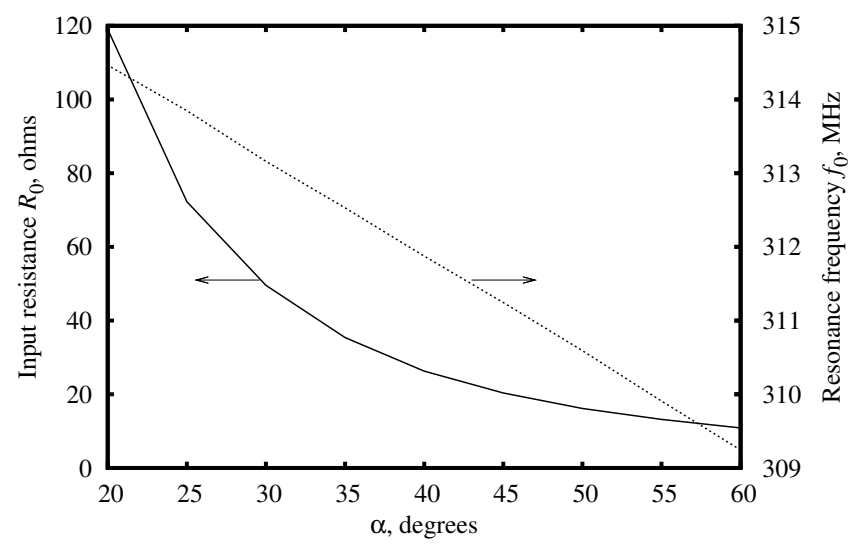

Fig. 10. Input resistance at resonance and the resonance frequency of the SSR antenna as a function of the excitation monopole length. $\gamma=4.9^{\circ}$.

The SSR antenna yields the ratio $Q / Q_{\mathrm{LB}}$ comparable to that of the MSH antenna (Section III), and at the same time, it can be used on a ground plane. Furthermore, among all presented antennas it exhibits the best characteristics in terms of radiation purity of the $\mathrm{TE}_{10}$ mode.

\section{B. Measured Results}

To facilitate the fabrication of an SSR antenna prototype, the number of split rings was reduced to $N_{\mathrm{SR}}=17$, and the

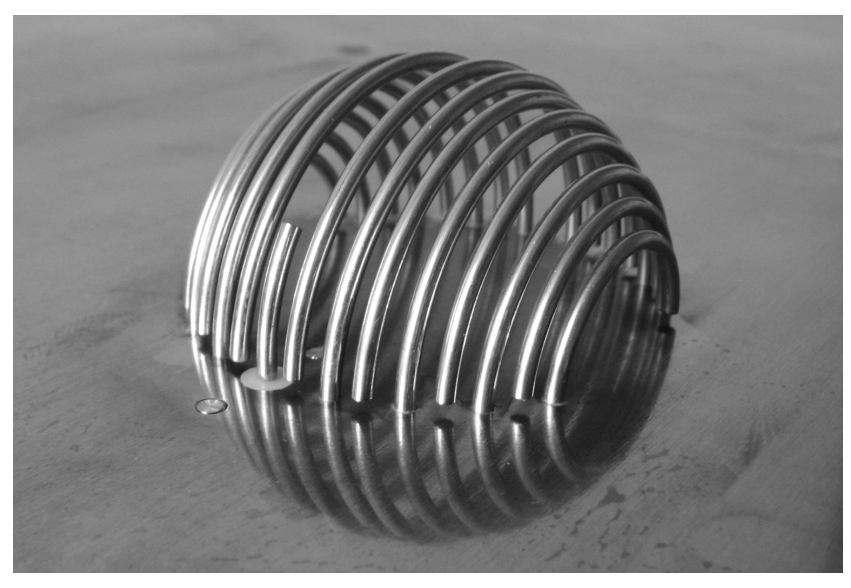

Fig. 11. Fabricated prototype the SSR antenna.

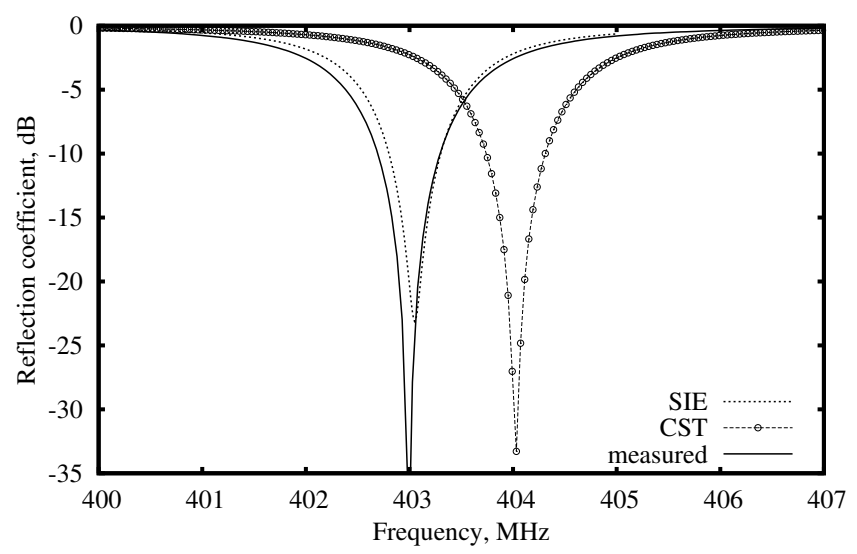

Fig. 12. Predicted and measured reflection coefficient of the manufactured SSR antenna.

wire of diameter $1.63 \mathrm{~mm}$ was selected. With the excitation monopole length $\alpha=33^{\circ}$, the SIE simulations predict the input impedance $R_{0}=43 \mathrm{ohms}$ and the resonance frequency $f_{0}=403 \mathrm{MHz}$, which corresponds to the electrical size $k a=0.184$. A supplementary simulation taking into account finite conductivity (copper) of the wires was carried out in the commercially available CST Microwave Studio, and the results are consistent with the SIE predictions. The fabricated prototype (Fig. 11) measured on a $1.5 \mathrm{~m}$ circular ground plane yields the input impedance $R_{0}=51 \mathrm{ohms}$ and the resonance frequency nearly coinciding with the SIE result, as illustrated in Fig. 12 and summarized in Table II.

Results of the radiation measurements performed at the DTU-ESA Spherical Near-field Antenna Test Facility are shown in Fig. 13 along with the radiation pattern obtained by the numerical simulations of the antenna on an infinite ground plane (note a change in the coordinate system). The effect of the finite-size ground plane is immediately recognized. The radiation pattern exhibits almost perfect left-right symmetry with a deep null in the crosspolarization at the boresight.

\section{CONCLUSION}

Three novel electrically small self-resonant antennas radiating the $\mathrm{TE}_{10}$ spherical mode are presented. The antennas are named after the spherical resonators they are based on: 
TABLE II

CHARACTERISTICS THE MANUFACTURED SRR ANTENNA.

\begin{tabular}{lcccccc}
\hline & $f_{0}, \mathrm{MHz}$ & $R_{0}, \Omega$ & efficiency & $Q$ & $Q_{\mathrm{LB}}$ & $Q / Q_{\mathrm{LB}}$ \\
\hline simulated (SIE)* & 403.0 & 43 & $100 \%$ & 564.0 & 165.6 & 3.41 \\
simulated (CST)** $^{*}$ & 404.0 & 52 & $76 \%$ & 462.3 & 124.9 & 3.70 \\
measured*** $^{*}$ & 403.0 & 51 & $73 \pm 2 \%$ & 442.9 & 120.9 & 3.66 \\
\hline
\end{tabular}

* PEC wires; infinite PEC ground plane.

*** copper wires; infinite PEC ground plane.

**** $1.5 \mathrm{~m}$ circular ground plane.

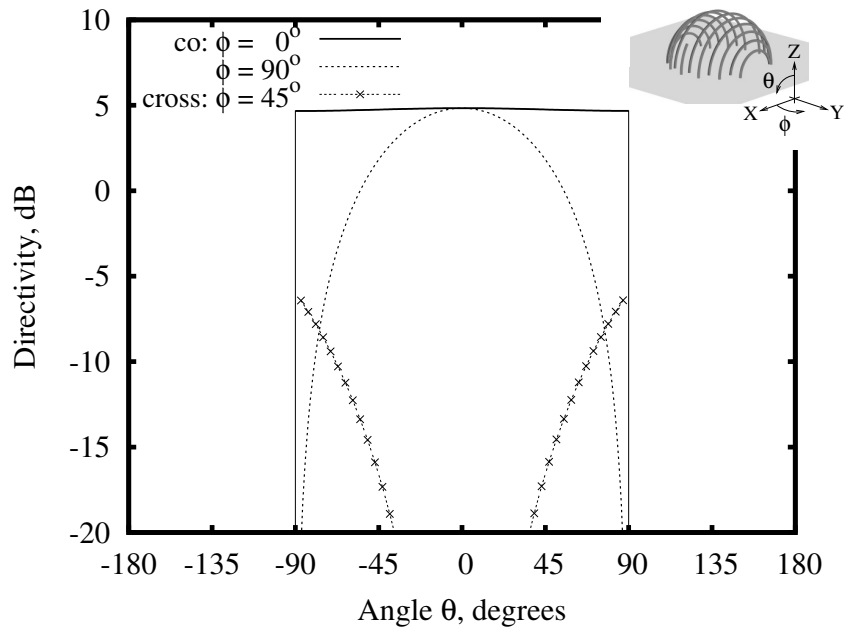

(a)

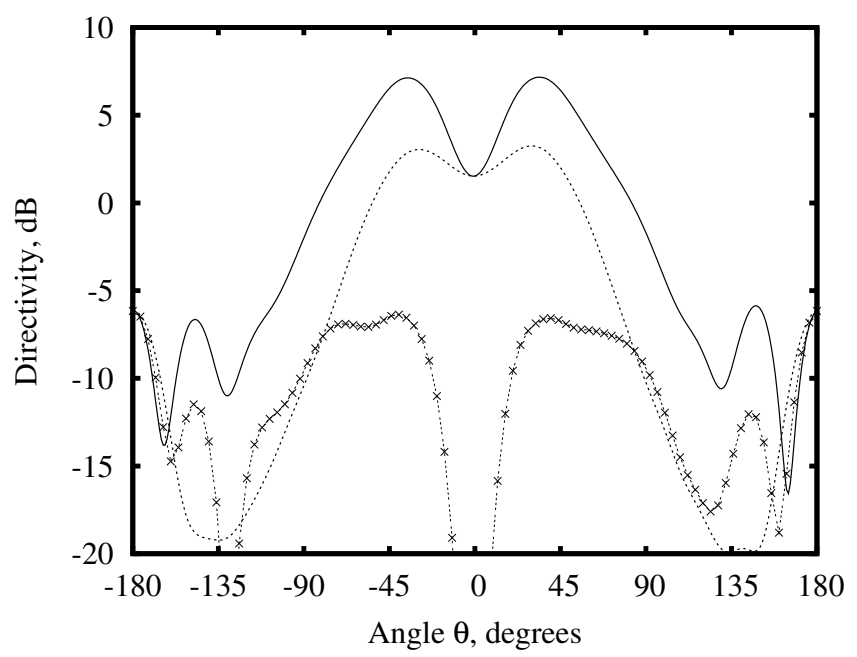

(b)

Fig. 13. Radiation pattern of the SSR antenna at $f_{0}=403 \mathrm{MHz}$ : (a) predicted, on an infinite ground plane; (b) measured, on a $1.5 \mathrm{~m}$ circular ground plane.

- multiarm spherical helix (MSH) antenna;

- spherical split-ring resonator (S-SRR) antenna;

- spherical split ring (SSR) antenna.

Theoretically, each of the resonators can be made arbitrarily small at a given frequency. The lower limit is set by a required frequency bandwidth. Each antenna is excited by a curved dipole (or monopole) located in the antenna equatorial plane. The antenna input resistance at resonance is a function of the excitation dipole (monopole) length. Thus, it is easily adjusted to match the antenna to a given feed line, no matter
TABLE III

CHARACTERISTICS THE TE 10 ANTENNAS.

\begin{tabular}{lcccc}
\hline antenna & $Q / Q_{\mathrm{LB}}$ & $\mathrm{TM}_{11}, \mathrm{~dB}$ & $\mathrm{TM}_{20}, \mathrm{~dB}$ & ground plane \\
\hline MSH & $<3.3$ & $<-30$ & -23 & no \\
S-SRR & 3.4 & $<-13$ & suppressed & yes \\
SSR & $<3.3$ & $<-32$ & suppressed & yes \\
\hline
\end{tabular}

how small the antenna is, and without any extra matching network. Moreover, this can be done nearly independent of the resonance frequency tuning, which is accomplished by adjusting the spherical resonator geometrical parameters. In this paper, the antennas have an electrical size $k a<0.26$, and all are matched to $50 \mathrm{ohms}$. The characteristics of the antennas are summarized in Table III.

The presented numerical results show that all three antennas yield the radiation quality factor $Q$ close to 3.0 times the Chu lower bound, which agrees with the theoretical prediction for an ideal electrically small magnetic dipole antenna made by Wheeler in 1958.

\section{ACKNOWLEDGMENT}

The author thanks Frank Persson and Dr. S. Pivnenko, both from the Technical University of Denmark, for fabrication and measurements of the SSR antenna prototype.

\section{REFERENCES}

[1] H. A. Wheeler, "Fundamental limitations of small antennas," Proc. IRE, vol. 35, no. 12, pp. 1479-1484, 1947.

[2] L. J. Chu, "Physical limitations of omni-directional antennas," J. Appl. Phys., vol. 19, no. 12, pp. 1163-1175, 1948.

[3] R. F. Harrington, J. Research National Bureau of Standards-D, Radio Propag., vol. 64D, no. 1, pp. 1-12, 1960.

[4] R. Collin and S. Rothschild, "Evaluation of antenna Q," IEEE Trans. Antennas Propagat., vol. 12, no. 1, pp. 23-27, Jan. 1964

[5] R. L. Fante, "Quality factor of general ideal antennas," IEEE Trans. Antennas Propagat., vol. 17, no. 2, pp. 151-155, Mar. 1969.

[6] J. S. McLean, "A re-examination of the fundamental limits on the radiation Q of electrically small antennas," IEEE Trans. Antennas Propagat., vol. 44, no. 5, pp. 672-676, May 1996.

[7] H. L. Thal, "New radiation Q limits for spherical wire antennas," IEEE Trans. Antennas Propagat., vol. 54, no. 10, pp. 2757-2763, Oct. 2006.

[8] S. R. Best, "The radiation properties of electrically small folded spherical helix antennas," IEEE Trans. Antennas Propagat., vol. 52, no. 4, pp. 953-960, Apr. 2004.

[9] _ - "Low Q electrically small linear and elliptical polarized spherical dipole antennas," IEEE Trans. Antennas Propagat., vol. 53, no. 3, pp. 1047-1053, Mar. 2005.

[10] H. A. Wheeler, "The spherical coil as an inductor, shield, or antenna," Proc. IRE, vol. 46, no. 9, pp. 1595-1602, 1958.

[11] S. R. Best, "A low Q electrically small magnetic (TE mode) dipole," IEEE Antennas Wireless Propagat. Lett., vol. 8, pp. 572-575, 2009.

[12] O. S. Kim and O. Breinbjerg, "Miniaturised self-resonant split-ring resonator antenna," Electron. Lett., vol. 45, no. 4, pp. 196-197, Feb. 2009. 
[13] — "Miniaturized planar split-ring resonator antenna," in Proc. IEEE Antennas Propag. Soc. Int. Symp., Charleston, South Carolina, June 1-5 2009.

[14] O. S. Kim, O. Breinbjerg, and A. D. Yaghjian, "Electrically small magnetic dipole antennas with quality factors approaching the Chu lower bound," IEEE Trans. Antennas Propagat., in press.

[15] E. Jørgensen, J. L. Volakis, P. Meincke, and O. Breinbjerg, "Higher order hierarchical Legendre basis functions for electromagnetic modeling," IEEE Trans. Antennas Propagat., vol. 52, no. 11, pp. 2985-2995, Nov. 2004.

[16] A. D. Yaghjian and S. R. Best, "Impedance, bandwidth, and Q of antennas," IEEE Trans. Antennas Propagat., vol. 53, no. 4, pp. 12981324, Apr. 2005.

[17] R. Marqués, F. Medina, and R. Rafii-El-Idrissi, "Role of bianisotropy in negative permeability and left-handed metamaterials," Phys. Rev. B, vol. 65, no. 14, pp. 144 440/1-6, Apr. 2002.

[18] P. Gay-Balmaz and O. J. F. Martin, "Efficient isotropic magnetic resonators," Appl. Phys. Lett., vol. 81, no. 5, pp. 939-941, Jul. 2002.

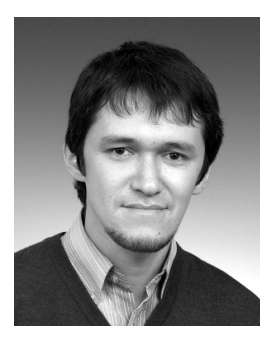

Oleksiy S. Kim received the M.S. degree and the $\mathrm{Ph} . \mathrm{D}$. degree from the National Technical University of Ukraine, Kiev, in 1996 and 2000, respectively, both in electrical engineering. In 2000, he joined the Antenna and Electromagnetics Group at the Technical University of Denmark (DTU). He is currently an associate professor with the Department of Electrical Engineering, ElectroScience Section, DTU.

His research interests include computational electromagnetics, metamaterials, electrically small antennas, photonic bandgap and plasmonic structures. 